\title{
FUNGOS MICORRÍZICOS ARBUSCULARES EM UM LATOSSOLO VERMELHO SOB MANEJOS E USOS NO CERRADO(1)
}

\author{
Dorotéia Alves Ferreira $^{(2)}$, Marco Aurélio Carbone Carneiro ${ }^{(3)} \&$ \\ Orivaldo José Saggin Junior ${ }^{(4)}$
}

\begin{abstract}
RESUMO
As modificações nas comunidades de fungos micorrízicos arbusculares (FMAs) induzidas por mudança de uso do solo e por diferentes usos agrícolas ainda são insuficientemente estudadas, particularmente no Cerrado. $O$ presente estudo teve como objetivo avaliar como a interferência antrópica pelo manejo e mudança de uso do solo atua sobre a densidade de esporos e diversidade dos FMAs em um Latossolo Vermelho distroférrico de Cerrado. O estudo avaliou cinco áreas: Mata Ciliar (MC), Borda da Mata (BM), Pastagem (Past), Plantio Direto (PD) e Área Desmatada (AD). Foram retirados 20 pontos amostrais, em delineamento inteiramente casualizado, para avaliação da densidade de esporos e diversidade de FMAs. Cada ponto amostral representava uma área de $250 \mathrm{~m}^{2}$ dentro de cada área. A área com o sistema de PD e a AD apresentaram menor densidade de esporos recuperados e baixa colonização micorrízica em relação às demais áreas devido à baixa quantidade de plantas vegetando nelas. As famílias de FMAs que apresentaram maior ocorrência nas áreas estudadas foram Acaulosporaceae, Glomeraceae e Gigasporaceae, sendo as duas primeiras dominantes nos ambientes menos antropizados de MC e BM. As espécies de FMAs de maior ocorrência foram Acaulospora scrobiculata, Glomus macrocarpum e Acaulospora tuberculata; as duas primeiras apareceram em todas as áreas, e a terceira apenas não ocorreu na área de PD. Essas espécies mostraram grande capacidade de adaptação aos ambientes alterados. As espécies Acaulospora rehmii, Acaulospora sp.3, Glomus etunicatum, Glomus tortuosum, Glomus sp.1, Gigaspora sp.2 e Scutellospora heterogama apresentaram baixa ocorrência, sendo recuperadas somente em uma das áreas estudadas. A área com maior densidade e ocorrência de espécies foi a Past, com 414 esporos (indivíduos) e 11 espécies. As áreas extremas quanto à diversidade de Shannon (H') foram a Past e a BM, com maior e menor índices, respectivamente. A análise de componentes
\end{abstract}

\footnotetext{
(1) Projeto Financiado pelo CNPq e CAPES. Recebido para publicação em 28 de setembro de 2010 e aprovado em 13 de novembro de 2011.

(2) Discente do Programa de Pós-Graduação em Agronomia da Universidade Federal de Goiás - UFG. BR 364, km 192, CEP 75804020 Jataí (GO). Bolsista CAPES. E-mail: dorotyferreita@yahoo.com.br

(3) Professor Associado, UFG. Bolsista CNPq. E-mail: carbonecarneiro@pq.cnpq.br

(4) Pesquisador A, Embrapa Agrobiologia, BR 465, Km 7, CEP 23890-000 Seropédica (RJ). E-mail: saggin@cnpab.embrapa.br
} 
principais indicou a formação de três grupos, sendo o primeiro formado pela MC e BM; o segundo, por AD e PD; e o terceiro, somente por Past. Conclui-se que a mudança no uso do solo promove alterações na comunidade de FMAs, podendo a densidade e diversidade de esporos ser ampliadas, no caso da pastagem, ou reduzidas, no caso de desmatamento.

Termos de indexação: densidade, diversidade, colonização radicular, pastagem, plantio direto, mata ciliar.

\section{SUMMARY: ARBUSCULAR MYCORRHIZAL FUNGI IN AN OXISOL UNDER MANAGEMENTS AND USES IN CERRADO}

The alterations in the communities of arbuscular mycorrhizal fungi (AMF) induced by changes in land use and different agricultural uses are still insufficiently studied, particularly in the Cerrado biome. This study evaluated how human interference by management and land use change affect the AMF density and diversity in a Cerrado Oxisol. The study evaluated five areas: Riparian Forest (MC), Riparian Forest Edge (BM), Pasture (Past), no-till monoculture (PD) and Riparian Deforested Area (AD). In each area, 20 plots of $250 \mathrm{~m} 2$ were marked. Within each plot, 10 subsamples were randomly collected to form a composite soil sample. The density and diversity of AMF spores, by morphological characteristics, were determined for each composite sample. In the PD and AD areas, the density of recovered spores and mycorrhizal colonization were lower than in the other areas due to the low density of living plants. The AMF families with highest abundance in the study areas were Acaulosporaceae, Glomeraceae and Gigasporaceae, the first two dominant in the areas with leass anthropogenic influence $M C$ and BM. The most frequent $A M F$ species were Acaulospora scrobiculata, Glomus macrocarpum and Acaulospora tuberculata, of which the first two appeared in all areas and the third was absent only in PD. These species have great capacity to adapt to changing environments. The occurrence of the species Acaulospora rehmii, Acaulospora sp.3, Glomus etunicatum, Glomus tortuosum, Glomus sp.1, Gigaspora sp.2 and Scutellospora heterogama was low, and were recovered in only one of the study areas. The area with highest density and species occurrence was Past with 414 spores (individuals) and 11 AMF species. The highest and lowest Shannon diversity $\left(H^{\prime}\right)$ index were calculated for Past and BM, respectively. Principal component analysis indicated the formation of three groups, the first with MC and BM, the second with PD and $A D$ and the third with only Past. It was concluded that land use changes modify the AMF community, which may increase spore density and diversity, as in the case of Pasture, or reduced, in the case of deforestation.

Index terms: spore density, diversity, root colonization, grassland, no-tillage, Riparian Forest.

\section{INTRODUÇÃO}

O bioma Cerrado é o segundo maior do território brasileiro e abrange um conjunto de ecossistemas (campos, savanas, matas e matas ciliares), os quais estão presentes na porção central do Brasil. Em várias áreas de Cerrado, incluindo áreas de nascente e matas ciliares, protegidas por força de lei, tem verificado-se grande devastação nas últimas décadas em função das atividades antrópicas, como a exploração agropecuária e as atividades de construção civil e mineração (Santos et al., 2009).

Ecossistema degradado é aquele que, após certo distúrbio, teve eliminada a vegetação e, junto, os meios bióticos. Assim, apresenta baixa resiliência, ou seja, o seu retorno ao estado anterior ao distúrbio pode não ocorrer, ou ser extremamente lento, necessitando da intervenção humana (Crestana et al., 2006). Isso tem trazido consequências ecológicas e ambientais bastante visíveis, as quais geram ações dos diversos setores da sociedade, principalmente no processo de recuperação/reabilitação dos atributos físicos, químicos e biológicos do solo.

O solo tem papel-chave em vários processos ambientais importantes, como o estabelecimento da vegetação, a atuação no ciclo hidrológico, no fluxo de água, no ciclo de vários nutrientes e energia nos ecossistemas (Moreira \& Siqueira, 2006). Além disso, o solo permite o estabelecimento de relações ecológicas diversas importantes para a 
biodiversidade macro e microscópica do ecossistema (Siqueira et al., 1994). Entre essas relações ecológicas destaca-se a micorriza, que é a simbiose mutualista formada entre raízes de plantas e fungos micorrízicos arbusculares (FMAs) representantes do filo Glomeromycota - classe Glomeromicetos. Esses fungos exercem grande influência na estruturação do solo e no crescimento, nutrição e adaptação das plantas aos ambientes, particularmente aqueles com estresses bióticos e abióticos no solo (Siqueira et al., 2007). O efeito das hifas fúngicas, principalmente dos fungos micorrízicos, na agregação do solo, tem recebido grande atenção, pois essa simbiose, além do seu papel na promoção nutricional de uma agricultura sustentável, promove a restauração ambiental (Siqueira et al., 1994).

Os FMAs são importantes componentes do sistema solo-planta, e sua evolução teria acontecido nos trópicos (Pirozynski, 1981) há mais de 460 milhões de anos, sendo possivelmente parceiros das plantas na evolução do ambiente aquático para o terrestre (Redecker et al., 2000). Assim, sua ocorrência é ubíqua no planeta, nas diversas ordens de plantas e ecossistemas terrestres (Brundrett, 2002; Stürmer \& Siqueira, 2008), sendo então considerada uma simbiose universal (Nicolson, 1967). Em razão da importância dos FMAs nos ecossistemas, presume-se que o aumento da riqueza de espécies desses fungos possa proporcionar incremento no crescimento ou na diversidade das plantas, com benefícios para ambos os parceiros (Klironomos et al., 2000). A ocorrência e a densidade dos FMAs dependem das características da planta hospedeira, do fungo e de fatores edafoclimáticos (Staddon et al., 2003) e antrópicos, por meio da pressão exercida pelo manejo e uso dos solos (Johnson \& Pfleger, 1992; Sylvia \& Williams, 1992).

Vários estudos demonstram a importância de conhecer a comunidade de FMAs para determinar estratégias de melhor manejo e uso do solo, aumento da eficiência no uso de fertilizantes e sua recuperação no caso de áreas degradadas (van der Heidjen et al., 1998; Klironomos et al., 2000; Allen et al., 2003). Em comparação com áreas preservadas, a comunidade de fungos micorrízicos pode ser reduzida em solos sem vegetação sob pousio (Thompson, 1987), nos inundados (Feldmann, 1994) e nos alterados pela agricultura (Cordeiro et al., 2005), envolvendo manejo inadequado ou culturas com baixa ou nenhuma dependência micorrízica, como é o caso das Brassicaceae (Kuyper et al., 2004).

Segundo Abbott \& Robson (1994) e Bever et al. (1996), o uso do solo, a cultura utilizada e o sistema de manejo empregado são fatores que influenciam a ocorrência e a abundância relativa das espécies de FMAs nos ecossistemas. Estudo realizado numa floresta da Costa Rica indica que o desmatamento reduziu a densidade e a diversidade de FMAs e que a conversão dessas áreas em pastagem promoveu alterações na comunidade desses fungos (Picone, 2000).

A ocorrência, a diversidade e o potencial simbiótico de comunidades de FMAs indígenas nos ecossistemas brasileiros são praticamente desconhecidos, considerando a extensão do território nacional e os poucos estudos em ecossistemas específicos (Schenck et al., 1989; Miranda \& Miranda, 1997; Stürmer \& Siqueira, 2008). As modificações na comunidade induzidas por mudança de uso do solo e por diferentes usos agrícolas também são insuficientemente estudadas, particularmente no Cerrado, encontrando-se poucos estudos nesse bioma (Cordeiro et al., 2005; Miranda, 2008).

Dessa forma, o objetivo deste estudo foi avaliar como a interferência antrópica pelo manejo e mudança de uso do solo atua sobre a densidade e diversidade dos fungos micorrízicos arbusculares (FMAs) em um Latossolo Vermelho distroférrico de Cerrado.

\section{MATERIAL E MÉTODOS}

O estudo foi realizado em áreas localizadas no município de Jataí/GO, próximo à cabeceira do rio Claro ( $17^{\circ} 56^{\prime} 35^{\prime \prime} \mathrm{S}, 51^{\circ} 43^{\prime} 38^{\prime \prime} \mathrm{W}$ e $672 \mathrm{~m}$ de altitude), em área sob interferência de uma pequena central hidrelétrica. A região apresenta temperatura média anual de $22{ }^{\circ} \mathrm{C}$ e precipitação pluvial média anual variando de 1.650 a $1.800 \mathrm{~mm}$, distribuída nos meses de setembro a abril; no mês de agosto de 2008 houve precipitação pluvial de $10 \mathrm{~mm}$. O solo foi classificado com Latossolo Vermelho distroférrico.

O estudo consistiu na comparação de áreas com distintos sistemas de manejo e uso do solo, sendo: Mata Ciliar (MC), Borda da Mata (BM), Pastagem (Past), Plantio Direto (PD) e Área Desmatada (AD), cujas descrições, histórico e coordenadas geográficas encontram-se no quadro 1 . Todos os sistemas de manejo e uso do solo estudado localizam-se próximos e sobre uma mesma mancha de solo, de forma que as condições ambientais foram homogêneas. Por se tratar de um estudo ecológico em áreas naturais com diferentes manejos e usos do solo já estabelecidos previamente ao presente estudo, não havia a possibilidade de aleatorização dessas áreas dentro de um delineamento estatístico planejado. Sob orientação de estatísticos, optou-se por casualizar os pontos de amostragem dentro de cada área e testar, antes da análise de variância, se havia homogeneidade de variância entre elas. Como isso foi confirmado, os dados foram analisados como 
um delineamento inteiramente casualizado com 20 pontos amostrais (pseudorrepetições). Cada ponto amostral representava uma área de $250 \mathrm{~m}^{2}$ dentro de cada área com sistema de manejo e uso do solo.

No interior de cada ponto amostral foram coletadas 10 subamostras em zigue-zague, com auxílio de um enxadão, na profundidade de $0-20 \mathrm{~cm}$, para formar uma amostra composta de $500 \mathrm{~g}$ de solo. Após a coleta, as amostras de solo foram acondicionadas em caixas térmicas e encaminhadas ao laboratório, onde foram peneiradas (malha de $2 \mathrm{~mm}$ ), retirando-se as raízes, que foram armazenadas em álcool (70 \%) para avaliação da colonização micorrízica. Uma parte do solo peneirado foi enviada para análise química e a outra armazenada em geladeira a $4{ }^{\circ} \mathrm{C}$, para posterior extração dos esporos de FMAs, determinação do número de esporos e identificação das espécies de FMAs para quantificação da diversidade e riqueza desses fungos.

Os atributos químicos e físicos avaliados foram: $\mathrm{pH}$ em água 2:1; $\mathrm{Al}$ e Ca, extraídos por KCl $1 \mathrm{~mol} \mathrm{~L}^{-1}$; K e P, extraídos por Mehlich-1; teor de argila: $651,1 \mathrm{~g} \mathrm{~kg}^{-1}$; silte: $47,8 \mathrm{~g} \mathrm{~kg}^{-1}$; e areia: $301,1 \mathrm{~g} \mathrm{~kg}^{-1}$. Os teores de $\mathrm{P}$ foram considerados baixos (3 a $6 \mathrm{mg} \mathrm{dm}^{-3}$ ) e médias (6 a $\left.12 \mathrm{mg} \mathrm{dm}^{-3}\right)$, considerando o teor de argila, conforme Sousa \& Lobato (2004).

As raízes finas coletadas foram clarificadas e coloridas conforme o método de Koske \& Gemma (1989) e Grace \& Stribley (1991). Posteriormente, essas raízes foram dispostas em lâminas com glicerina e observadas em microscópio óptico, quantificando-se a colonização micorrízica em cerca de 100 a 150 segmentos de raiz com $1 \mathrm{~mm}$ de comprimento, amostrados aleatoriamente.

A extração dos esporos de FMAs foi realizada segundo Gerdemann \& Nicolson (1963), seguida de centrifugações em água e sacarose (45\%). Os esporos foram contados e posteriormente separados segundo suas características morfológicas de cor e tamanho, sendo armazenados congelados a $-20^{\circ} \mathrm{C}$ em tubos Eppendorf. Os esporos de cada tubo Eppendorf foram vertidos sobre uma placa de Petri e divididos em dois grupos, os quais, com uma pipeta de Pasteur, foram transferidos para pontos sobre uma lâmina de vidro, para microscopia. Nessa, um dos grupos foi fixado com PVLG (álcool polivinílicolactoglicerol) e outro com PVLG + reagente de Melzer (1:1) (Morton, 1993). Os esporos fixados com PVLG + reagente de Melzer foram submetidos a uma pequena pressão sobre a lamínula, para rompimento das paredes, o que permitiu a coloração das paredes internas, servindo de parâmetro de identificação. Após a montagem, as lâminas foram mantidas em temperatura ambiente, para secagem inicial, e posteriormente levadas à estufa $\left( \pm 40^{\circ} \mathrm{C}\right)$, para secagem completa do PVLG.

Aidentificação dos FMAs foi realizada na Embrapa Agrobiologia, com auxílio de um microscópio óptico equipado com ocular micrométrica. Para subsidiar o trabalho de identificação, foram utilizados o manual

\section{Quadro 1. Histórico e descrição das áreas em estudo}

\section{Área}

\section{Descrição}

Mata ciliar com espécies arbóreas e arbustivas, característica do bioma do Cerrado,

Mata Ciliar (MC) apresentando grande quantidade de serapilheira. Ocupa uma faixa de $200 \mathrm{~m}$ de largura e $3.000 \mathrm{~m}$ de comprimento, disposta paralelamente ao rio Claro. Localizada a $17^{\circ} 55^{\prime} 35^{\prime}$ ' $\mathrm{S}$ e $51^{\circ} 43^{\prime} 26^{\prime \prime} \mathrm{W}$.

Borda da Mata (BM)

Área de transição entre a Mata Ciliar e a Pastagem, localizada no entorno desta. Vegetada com espécies arbóreas típicas de mata ciliar do Cerrado e com B. brizantha pastejada por gado bovino. Localizada a $17^{\circ} 55^{\prime} 35^{\prime \prime} \mathrm{S}$ e $51^{\circ} 43^{\prime} 25^{\prime \prime} \mathrm{W}$.

Pastagem (Past)

Área sob pastagem de B. brizantha desde 1996. Em 2006 foram aplicadas 2,5 t de calcário (PRNT $65 \%$ ) na superfície. Apresenta sinais de degradação do solo, com sulcos de erosão, solo descoberto e depauperação da fertilidade do solo. Ocupa uma área de 20 ha. Localizada a $17^{\circ} 56^{\prime} 23^{\prime \prime} \mathrm{S}$ e $51^{\circ} 43^{\prime} 36^{\prime \prime} \mathrm{W}$.

Área de agricultura no sistema de plantio direto desde 2000, utilizando a sucessão soja/ milho ou soja/sorgo. Na safra 2007/08 o plantio de soja foi realizado em outubro, utilizando $360 \mathrm{~kg} \mathrm{ha}^{-1}$ do fertilizante formulado 0-23-23 (NPK), e na safrinha, plantada em fevereiro, sorgo, utilizando $150 \mathrm{~kg} \mathrm{ha}^{-1}$ de 08-20-10 (NPK). Na época da amostragem a área encontravase em pousio, vegetada com plantas invasoras. Ocupa uma área de 45 ha. Localizada a $17^{\circ} 55^{\prime} 41^{\prime \prime} \mathrm{S}$ e $51^{\circ} 43^{\prime} 20^{\prime \prime} \mathrm{W}$.

Área sob interferência da represa de uma pequena central hidrelétrica. Em maio de 2008 foi retirada toda a mata ciliar em razão do enchimento da represa. Posteriormente, será revegetada e formada nova mata ciliar, respeitando a zona de alagamento da represa. $\mathrm{Na}$ época da amostragem, a área estava vegetada com plantas invasoras rasteiras. Ocupa uma área em torno de 80 ha. Localizada a $17^{\circ} 56^{\prime} 14^{\prime \prime} \mathrm{S}$ e $51^{\circ} 43^{\prime} 45^{\prime \prime} \mathrm{W}$.

Área Desmatada (AD) 
de Schenck \& Pérez (1990), artigos originais da descrição das espécies e descrições das espécies fornecidas no site da "International Culture Collection of Arbuscular and Vesicular-Arbuscular Mycorrhizal Fungi” (INVAM, 2009).

Os índices ecológicos foram calculados utilizando o número de espécies recuperadas $(\mathrm{S})$ em cada área em $50 \mathrm{~mL}$ de solo; a riqueza de espécies foi estimada pelo número total de indivíduos de uma espécie em cada área; e o índice de diversidade de Shannon foi calculado por $\mathrm{H}^{\prime}=\sum_{\mathrm{i}} \mathrm{p}_{\mathrm{i}}\left(\log \mathrm{p}_{\mathrm{i}}\right)$, em que $\mathrm{p}_{\mathrm{i}}$ foi a abundância de esporos recuperados de cada espécie em relação ao total de (i) espécies de FMAs encontradas (Odum, 1988). Todos esses índices e as diferenças de diversidade entre as áreas foram calculados utilizando o programa estatístico PAST (Hammer et al., 2001).

Para auxiliar na interpretação dos dados, os resultados foram submetidos à análise de componentes principais, envolvendo as variáveis referentes aos FMAs e químicas do solo $(\mathrm{pH}$, $\mathrm{Ca}, \mathrm{Al}, \mathrm{K}$ e P). Para isso, o conjunto dos dados foi reduzido em combinações lineares, gerando escores das duas primeiras variáveis, que explicam mais de $80 \%$ da variação total. Posteriormente, procedeu-se ao agrupamento das áreas pelo método de agrupamento de Tocher, por meio da matriz de distância generalizada de Mahalanobis (Cruz \& Regazzi, 1994), utilizando o programa Genes (Cruz, 1997).

Os resultados de densidade de esporos, colonização micorrízica, características químicas do solo e índice de diversidade de Shannon $\left(\mathrm{H}^{\prime}\right)$, após verificação da homogeneidade de variância entre as áreas estudadas, foram submetidos à análise de variância, e as médias foram comparadas pelo teste de Tukey a $5 \%$ e por correlação de Pearson, utilizando o pacote estatístico SAEG (SAEG, 2007).

\section{RESULTADOS E DISCUSSÃO}

A densidade de esporos de fungos micorrízicos arbusculares (FMAs) e a colonização micorrízica apresentaram variações entre as áreas estudadas, encontrando-se maior densidade e colonização micorrízica $(p \leq 0,05)$ nas áreas sob Pastagem, Mata Ciliar e Borda da Mata, diferindo da área de Plantio Direto (Quadro 2).

Os atributos químicos $(\mathrm{pH}, \mathrm{Al}, \mathrm{Ca}, \mathrm{K}$ e $\mathrm{P})$ das áreas estudadas encontram-se no quadro 2.

Esses fungos, por serem biotróficos obrigatórios, necessitam de plantas (raízes) para sua sobrevivência (Moreira \& Siqueira, 2006), e as áreas de Plantio Direto (PD) e desmatada (AD), na ocasião da amostragem, apresentavam-se com baixa quantidade de vegetação, o que pode ter provocado a redução de propágulos viáveis, como os esporos, alterando diretamente a colonização radicular.

A produção de esporos de FMAs é um mecanismo de perpetuação das espécies, sendo estimulada quando a planta e o fungo são submetidos a algum estresse (Johnson \& Pfleger, 1992; Guadarrama \& Álvarez-Sánches, 1999; Miranda, 2008). No momento da amostragem, as áreas estavam submetidas a forte estresse hídrico, o que pode ter contribuído para aumentar a densidade de esporos nas áreas com presença de vegetação, como na Mata Ciliar, na Borda da Mata e na Pastagem. Nas áreas sob Plantio Direto e Área Desmatada, a cobertura vegetal foi estimada em cerca de 3 e $0,5 \mathrm{Mg} \mathrm{ha}^{-1}$ de matéria seca, respectivamente, composta de vegetação espontânea e rasteira. Isso alterou negativamente a densidade de esporos nas áreas $\mathrm{PD}$ e $\mathrm{AD}$ e a colonização micorrízica somente na área de PD. Outro fator importante foi a alta correlação, negativa e significativa, entre o $\mathrm{P}$ no solo e a produção de esporos $\left(r=-0,85^{* *}\right)$ e a de $\mathrm{P}$ no solo e colonização micorrízica $\left(r=-0,88^{* *}\right)$. Os

Quadro 2. Densidade de esporos (DEN) de fungos micorrízicos arbusculares nativos, colonização micorrízica (CM) das raízes das plantas hospedeiras e atributos químicos avaliados nas áreas de estudo

\begin{tabular}{|c|c|c|c|c|c|c|c|}
\hline Área & DEN & $\mathbf{C M}$ & pH & Al & $\mathrm{Ca}$ & $\mathbf{K}$ & $\mathbf{P}$ \\
\hline & $\mathrm{N}^{\circ} 50 \mathrm{~mL}$ de solo $^{-1}$ & $\%$ & Em água & \multicolumn{2}{|c|}{$-\mathrm{cmol}_{\mathrm{c}} \mathrm{dm}^{-3}$} & \multicolumn{2}{|c|}{$\mathrm{mg} \mathrm{dm}^{-3}$} \\
\hline $\mathrm{MC}$ & $63 \mathrm{a}$ & $51 \mathrm{a}$ & $5,6 \mathrm{a}$ & $0,09 \mathrm{a}$ & $6,27 \mathrm{~b}$ & $241 \mathrm{~b}$ & $1,36 \mathrm{c}$ \\
\hline BM & $62 \mathrm{a}$ & $51 \mathrm{a}$ & $5,7 \mathrm{a}$ & $0,07 \mathrm{a}$ & $5,75 \mathrm{~b}$ & $338 \mathrm{a}$ & $4,74 \mathrm{~b}$ \\
\hline Past & $83 \mathrm{a}$ & $62 \mathrm{a}$ & $5,7 \mathrm{a}$ & $0,12 \mathrm{a}$ & $2,44 \mathrm{~d}$ & $93 \mathrm{c}$ & $0,85 \mathrm{c}$ \\
\hline $\mathrm{PD}$ & $33 \mathrm{~b}$ & $40 \mathrm{~b}$ & 5,9 a & $0,03 \mathrm{a}$ & $7,26 \mathrm{a}$ & $325 \mathrm{a}$ & $9,10 \mathrm{a}$ \\
\hline $\mathrm{AD}$ & $42 \mathrm{~b}$ & $49 \mathrm{ab}$ & $5,9 \mathrm{a}$ & $0,04 \mathrm{a}$ & $4,10 \mathrm{c}$ & $110 \mathrm{c}$ & $4,23 \mathrm{~b}$ \\
\hline CV ( \%) & 37 & 23 & 12 & 8 & 14 & 20 & 19 \\
\hline
\end{tabular}

Áreas: MC: Mata Ciliar; BM: Borda da Mata; Past: Pastagem; PD: Plantio Direto; AD: Área Desmatada. Médias seguidas de mesma letra na coluna não diferem entre si pelo teste de Tukey a $5 \%$. CV: coeficiente de variação. 
outros atributos químicos do solo, como $\mathrm{pH}, \mathrm{Al}, \mathrm{Ca}$ e $K$, não mostraram correlação com as variáveis micorrízicas (densidade e colonização micorrízica). Segundo Sieverding (1990), em agroecossistemas com manejo intensivo ocorre redução da esporulação dos FMAs, quando comparados com ecossistemas naturais. Esse fato já foi observado por Carneiro et al. (2009) e Miranda (2008) em estudos realizados no bioma Cerrado submetido a diferentes intensidades de manejo e uso do solo.

As famílias de FMAs que apresentaram maior ocorrência nas áreas estudadas foram Acaulosporaceae e Glomeraceae, seguidas de Gigasporaceae; a de menor ocorrência em todas as áreas foi Archaeosporaceae (Quadro 3).

Observou-se ainda que, entre as famílias de FMAs encontradas, a que apresentava maior número de espécies foi Acaulosporaceae (Acaulospora rehmii, Acaulospora scrobiculata, Acaulospora tuberculata, Acaulospora sp.1, Acaulospora sp.2 e Acaulospora sp.3), e o menor número foi da família Archaeosporaceae, observando-se apenas uma espécie (Archaeospora leptoticha).

Nas áreas de Mata Ciliar, Borda da Mata e Plantio Direto há predominância de espécies da família Acaulosporaceae e Glomeraceae. Já na área de Pastagem e na Área Desmatada foram encontradas espécies das famílias Acaulosporaceae, Glomeraceae e Gigasporaceae em similar proporção (Quadro 3). Na área de Plantio Direto, embora tenham predominado esporos de Acaulosporaceae e Glomeraceae, a densidade de esporos foi menor que nas áreas de Mata Ciliar e Borda da Mata (Quadro 3).

A dominância das famílias Acaulosporaceae e Glomeraceae em áreas com intervenção antrópica foi verificada por Cuenca et al. (1998) e Boddington \& Dodd (2000); em áreas cultivadas na região de clima temperado, vários outros estudos também indicam a predominância dessas famílias (FrankeSnyder et al., 2001; Jansa et al., 2002; Oehl et al., 2004). Os resultados deste estudo corroboram com os encontrados por Miranda (2008) em diferentes solos de Cerrado nativo e cultivados, onde também notou-se a predominância das famílias Glomeraceae e Acaulosporaceae, respectivamente. Isso ocorre devido à maior capacidade de adaptação dessas famílias a solos submetidos a diferentes manejos e usos, como observado em diferentes estudos (Carrenho et al., 2002; Bennedeti et al., 2005; Silva Júnior \& Cardoso, 2006).

As espécies S. heterogama, A. tuberculata, G. macrocarpum e A. scrobiculata foram as que apresentaram maior densidade de esporos: em média, mais de 70 esporos em cada $50 \mathrm{~mL}$ de solo rizosférico (Quadro 4). A. scrobiculata e G. macrocarpum foram observadas em todas as cinco áreas de estudo, e A. tuberculata somente não foi encontrada na área de Plantio Direto. As espécies S. pellucida e Ar. leptoticha também apresentaram ocorrência generalizada nas áreas, sendo observadas em quatro delas, porém com número menor de esporos na rizosfera que as anteriores: em média, 13 e 9 esporos de FMAs por $50 \mathrm{~mL}$ de solo, respectivamente. S. pellucida somente não foi recuperada em solo sob Mata Ciliar, e Ar. leptoticha, na Borda da Mata (Quadro 4).

As espécies A. rehmii, Acaulospora sp.3, G. etunicatum, G. tortuosum, Glomus sp.1, Gigaspora sp. 2 e S. heterogama apresentaram baixa ocorrência nas áreas estudadas, ou seja, foram recuperadas somente em uma das áreas estudadas; $A$. rehmii e G. etunicatum, somente na área de Plantio Direto; Gigaspora sp.2, apenas na Área Desmatada; Glomus sp.1, somente na Borda da Mata; e Acaulospora sp.3, G. tortuosum e S. heterogama, em área de Pastagem. Entre essas espécies, apenas $S$. heterogama mostrou maior densidade de esporos recuperados: acima de 80 esporos por $50 \mathrm{~mL}^{-1}$ de solo (Quadro 4).

Nas áreas estudadas, foram recuperadas e observadas três espécies de Acaulospora não identificadas, duas de Gigaspora e uma espécie de Glomus. Há dificuldade para identificar as espécies de FMAs com a taxonomia morfológica, portanto a falta de identificação das espécies nem sempre indica a presença de espécies novas. Deve-se buscar

Quadro 3. Número de espécies de FMAs por famílias recuperadas no solo da Mata Ciliar (MC), Borda da Mata (BM), Pastagem (Past), Plantio Direto (PD) e Área Desmatada (AD)

\begin{tabular}{lllcll}
\hline Família & MC & BM & Past & PD & AD \\
\hline & & & $N^{\circ}$ de espécies & & $2(40)$ \\
Glomeraceae & $1(65)^{(1)}$ & $2(98)$ & $2(132)$ & $5(111)$ & $2(76)$ \\
Acaulosporaceae & $4(234)$ & $2(197)$ & $3(100)$ & $1(1)$ & $3(73)$ \\
Gigasporaceae & 0 & $1(12)$ & $1(4)$ & $1(12)$ & $1(6)$ \\
Archaeosporaceae & $1(13)$ & 0 & & \\
\hline
\end{tabular}

(1) Valores entre parênteses representam a quantidade total de esporos de FMAs em cada família. 
Quadro 4. Espécies de fungos micorrízicos arbusculares e respectivo número de esporos recuperados na Mata Ciliar (MC), Borda da Mata (BM), Pastagem (Past), Plantio Direto (PD) e Área Desmatada (AD)

\begin{tabular}{|c|c|c|c|c|c|}
\hline Espécies de FMAs & MC & $\mathbf{B M}$ & Past & PD & $\mathrm{AD}$ \\
\hline & \multicolumn{5}{|c|}{$\mathrm{n}^{\circ}$ de esporos $50 \mathrm{~mL}^{-1}$} \\
\hline Acaulospora rehmii Siev. \& Toro & - & - & - & 10 & - \\
\hline Acaulospora scrobiculata Trap. & 123 & 74 & 37 & 82 & 69 \\
\hline Acaulospora tuberculata Jan. \& Trap. & 65 & 123 & 91 & - & 4 \\
\hline Acaulospora sp. 1 & 27 & - & 19 & 5 & - \\
\hline Acaulospora sp. 2 & 19 & - & 17 & 14 & - \\
\hline Acaulospora sp. 3 & - & - & 14 & - & - \\
\hline Archaeospora leptoticha (Sch. \& Smi.) Mort. \& Red. & 13 & - & 4 & 12 & 6 \\
\hline Glomus etunicatum Beck. \& Gerd. & - & - & - & 6 & - \\
\hline Glomus macrocarpum Tul.\& Tul. & 65 & 92 & 126 & 34 & 59 \\
\hline Glomus tortuosum Sch. \& Smi. & - & - & 6 & - & - \\
\hline Glomus sp.1 & - & 6 & - & - & - \\
\hline Gigaspora sp.1 & - & - & 6 & - & 16 \\
\hline Gigaspora sp.2 & - & - & - & - & 32 \\
\hline Scutellospora heterogama (Nic. \& Ger.) Wal. \& Sand. & - & - & 81 & - & - \\
\hline Scutellospora pellucida (Nic. \& Sch.) Wal. \& Sand. & - & 12 & 13 & 1 & 25 \\
\hline
\end{tabular}

o isolamento das espécies não identificadas em culturas puras, a fim de possibilitar futuramente sua perfeita identificação ou mesmo descrição de nova espécie. Entre espécies não identificadas, as de Acaulospora apareceram nas áreas de Mata Ciliar, Pastagem e Plantio Direto; as de Gigaspora, na Área Desmatada e na Pastagem; e a de Glomus, somente na Borda da Mata.

A riqueza de espécies de FMAs variou entre as áreas estudadas (Quadro 5). No presente estudo foram encontradas, na área de Pastagem, 11 espécies (sete identificadas e quatro não identificadas); na área de Plantio Direto, oito espécies (seis identificadas e duas não identificadas); e na Área Desmatada, sete espécies (cinco identificadas e duas não identificadas), sendo esses números superiores aos das áreas com menor interferência antrópica e com presença de vegetação arbórea, como a Mata Ciliar e Borda da Mata, com seis (duas não identificadas) e cinco espécies (uma não identificada), respectivamente (Quadro 5). Áreas agrícolas e desmatadas apresentam redução na abundância e distribuição dos FMAs em relação à área não perturbada, conforme observado por Manson et al. (1992), Caproni et al. (2003) e Miranda et al. (2005) corroborando os resultados obtidos neste estudo.

Para a área de Pastagem, estudo conduzido por Treseder \& Cross (2006) constatou tendência de maior diversidade de FMAs quando comparada com áreas antropizadas, confirmando os resultados encontrados neste estudo. Isso pode ser devido a uma interação entre planta/fungo e pelo fato de as gramíneas possuírem alta eficiência fotossintética, mantendo o maior grau de infecção de todas as espécies de FMAs individualmente, além de terem sistema radicular ramificado e abundante, que diminui a competição entre espécies de FMAs por sítios de infecção (Klironomos et al., 2000).

$\mathrm{O}$ índice de diversidade de Shannon (H') apresentou variação entre as áreas estudadas (Quadro 5). Os maiores índices foram obtidos nas áreas de Pastagem, Mata Ciliar e Área Desmatada, e os menores, nas áreas de Plantio Direto e Borda da Mata. O índice de diversidade H' é considerado ideal quando o objetivo é estudar os efeitos das perturbações sofridas pelos ecossistemas, pois atribui maior peso às espécies não dominantes, consideradas espécies raras, que são as primeiras a sentir os efeitos dos impactos ambientais (Odum, 1988). Nesse sentido, a observação do maior índice para a área sob Pastagem reflete a maior riqueza de espécies recuperadas, fato que não ocorreu na Borda da Mata e Plantio Direto.

Considerando os atributos químicos do solo $(\mathrm{pH}$, $\mathrm{Ca}, \mathrm{Al}, \mathrm{Ke} \mathrm{P}$ ) e os biológicos relacionados aos FMAs, obteve-se, por meio da análise de componentes principais, uma variação total de $89,78 \%$, o que está de acordo com Cruz \& Regazzi (1994). Os atributos de menor contribuição para a variação dos dados foram o $\mathrm{Al}^{3+}$ e o $\mathrm{pH}$, e os de maior contribuição, o $\mathrm{P}$ e a densidade de esporos. Isso mostra que o $\mathrm{Al}^{3+}$ pouco contribui para separar as áreas estudadas e não possui relação com os resultados obtidos pela 
Quadro 5. Índice diversidade de Shannon (H'), número total de espécies encontradas em cada área (riqueza) e total de indivíduos recuperados nas diferentes áreas estudadas

\begin{tabular}{lcccc}
\hline Área & \multicolumn{1}{c}{$\mathbf{H}^{\prime}$} & Riqueza & Total de indivíduos $^{(1)}$ \\
\hline Mata Ciliar & 1,54 & ab & $\mathrm{n}^{\circ}$ espécies & $\mathrm{n}^{\circ}$ esporos \\
Borda da Mata & 1,27 & $\mathrm{c}$ & 6 & 312 \\
Pastagem & 1,89 & $\mathrm{a}$ & 11 & 307 \\
Plantio Direto & 1,50 & $\mathrm{~b}$ & 8 & 414 \\
Área Desmatada & 1,63 & $\mathrm{ab}$ & 7 & 211 \\
CV (\%) & 12 & & & 164 \\
\hline
\end{tabular}

(1) Refere-se ao total de esporos coletados nas 20 amostras por área. Médias seguidas pela mesma letra, na coluna, não diferem entre si pelo teste t a $5 \%$.

avaliação dos FMAs no presente estudo. Com base no método de agrupamento de Tocher, houve a formação de três grupos: o primeiro, formado pelas áreas de Mata Ciliar e Borda da Mata; o segundo, pela Área Desmatada e Plantio Direto; e o terceiro, somente pela área de Pastagem (Figura 1).

Esses resultados indicam que as áreas de Plantio Direto e Área Desmatada apresentam comportamento similar quanto aos atributos estudados. Eles também mostram que a Pastagem difere das demais áreas, certamente por favorecer a ocorrência de diferentes espécies de FMAs e propiciar dominância das espécies Acaulospora tuberculata, Glomus macrocarpum e Scutellospora heterogama, o que também foi observado por Mathimaram et al. (2007) em área sob Latossolo com a cultura do milho no Quênia. Jansa et al. (2002) observaram maior ocorrência de Acaulospora em áreas sem interferência antrópica, tal como no presente trabalho, em que a dominância de Acaulospora foi observada principalmente nas áreas de Mata Ciliar e Borda da Mata, onde o impacto antrópico foi menor que nas demais áreas que apresentavam perturbações mais profundas e recentes (Plantio Direto, Área Desmatada e Pastagem).

A mudança nas comunidades de FMAs promovida por manejos agrícolas intensivos pode levar à perda da efetividade simbiótica de promoção de crescimento vegetal, como observado em monoculturas de seringueira na Amazônia, comparada com áreas de sua ocorrência natural (Feldmann et al., 2002). Segundo esses autores, a diversidade de vegetação pode ser o fator que incrementa a heterogeneidade genética dos FMAs e mantém a efetividade simbiótica da comunidade.

Portanto, a comunidade de FMAs está intimamente relacionada ao solo (manejo e fertilidade) e à planta hospedeira (diversidade e fisiologia do crescimento), sendo essa comunidade um reflexo da qualidade dos ecossistemas e de grande importância para sua resiliência. A comunidade de FMAs mantida diversa permite melhor equilíbrio ecológico na rizosfera e, certamente, ajuda a manter a sustentabilidade dos solos. Práticas intensivas, como monocultura, mudança de uso do solo e desmatamento, impactam as comunidades de FMAs (Douds \& Millner, 1999), como observado neste estudo. A extensão desses impactos na comunidade de FMAs ainda precisa ser estudada quanto ao prejuízo na sustentabilidade dos sistemas e quanto à erosão genética causada a esses fungos ancestrais. Material genético de FMAs, como espécies raras, espécies ainda não identificadas ou cultivadas, ecótipos locais ou específicos para determinada planta, pode estar sendo perdido antes que a evolução da taxonomia molecular desvende sua real diversidade.

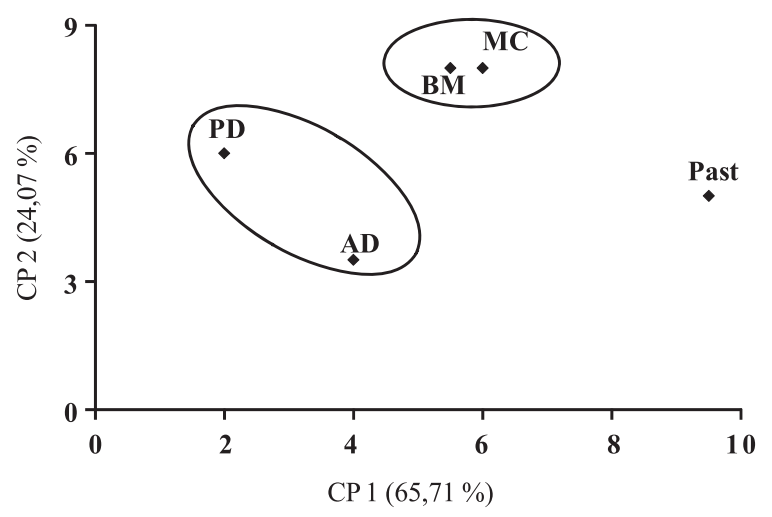

Figura 1. Relação entre a componente principal 1 (CP1) e a 2 (CP2) da análise de componentes principais (ACP), mostrando os agrupamentos (Tocher) das áreas mais semelhantes, com base nos atributos químicos (pH, Al, Ca, K e P) e densidade de esporos e diversidade de FMAs. 


\section{CONCLUSÕES}

1. A mudança no uso do solo promove alterações na comunidade de FMAs, podendo a densidade de esporos e diversidade dos fungos micorrízicos arbusculares ser ampliadas, no caso da pastagem, ou reduzidas, no caso de monoculturas ou desmatamento.

2. As famílias de fungos micorrízicos arbusculares de maior ocorrência nas áreas estudadas foram Acaulosporaceae, Glomeraceae e Gigasporaceae, sendo as duas primeiras preponderantes nos ambientes menos antropizados.

3. A Pastagem com braquiária promove maior diversidade e riqueza de esporos de FMAs que as demais áreas dominadas por dicotiledôneas.

4. As espécies A. scrobiculata, G. macrocarpum e A. tuberculata são dominantes nos ambientes estudados, tanto naqueles mais preservados quanto nos alterados por diferentes manejos.

\section{AGRADECIMENTOS}

Ao Conselho Nacional de Desenvolvimento Científico e Tecnológico $(\mathrm{CNPq})$, pelo auxílio financeiro (Processo 577559/2008-7) para execução do estudo e pela bolsa de pesquisa (Processo 305257/2008-0). À Capes, pela concessão de bolsa de mestrado à primeira autora. Ao Dr. Sidney Luiz Stürmer, pela revisão e contribuição para este estudo.

\section{LITERATURA CITADA}

ABBOTT, L.K. \& ROBSON, A.D. The impact of agricultural practices on mycorrhizal fungi. In: PANKHURST, C.E.; DOUBE, B.M.; GUPTA, V.V.S.R. \& GRACE, P.R., eds. Soil biota: Management in sustainable farming systems. Victoria, CISRO, 1994. p.88-95.

ALLEN, M.F.; SWENSON, W.; QUEREJETA, J.I.; EGERTONWARBURTON, L.M. \& TRESEDER, K.K. Ecology of mycorrhizae: A conceptual framework for complex interactions among plants and fungi. Ann. Rev. Phytopathol., 41:271-303, 2003.

BENNEDETI, T.; ANTONIOLLI, Z.I.; GIRACCA, E.M.N. \& STEFFEN, R.B. Diversidade de fungos micorrízicos arbusculares na cultura do milho após uso de espécies de plantas de cobertura de solo. R. Ci. Agrovet., 4:44-51, 2005.

BEVER, J.D.; MORTON, J.B.; ANTONOVICS, J. \& SCHULTZ, P.A. Host-dependent sporulation and species diversity of arbuscular mycorrhizal fungi in a mown grassland. J. Ecol., 84:71-82, 1996.
BODDINGTON, C.L. \& DODD, J.C. The effect of agricultural practices on the development of indigenous arbuscular mycorrhizal fungi. I. Field studies in Indonésia ultisol. Plant Soil, 218:137-144, 2000.

BRUNDRETT, M. Coevolution of roots and mycorrhizas of land plants. New Phytol., 154:275-304, 2002.

CAPRONI, A.L.; FRANCO, A.A.; BERBARA, R.L.L.; GRANHA, J.R.D.O.; SILVA, E.M.R. \& SAGGIN JÚNIOR, O.J. Capacidade infectiva de fungos micorrízicos arbusculares em áreas reflorestadas após mineração de bauxita no Pará. Pesq. Agropec. Bras., 38:937-945, 2003.

CARNEIRO, M.A.C.; SOUZA, E.D.; REIS, E.F.; PEREIRA, H.S. \& AZEVEDO, W.R. Atributos físicos, químicos e biológicos de solo de Cerrado sob diferentes sistemas de uso e manejo. R. Bras. Ci. Solo, 33:147-157, 2009.

CARRENHO, R.; TRUFEM, S.F.B. \& BONONI, V.L.R. Effects of using different host plants on the detected biodiversity of arbuscular mycorrhizal fungi from an agroecosystem. R. Bras. Bot., 25:93-101, 2002.

CORDEIRO, M.A.S.; CARNEIRO, M.A.C.; PAULINO, H.B. \& SAGGIN JÚNIOR, O.J. Colonização e densidade de esporos de fungos micorrízicos em dois solos do cerrado sob diferentes sistemas de manejo. Pesq. Agropec. Trop., 35:147-153, 2005.

CRESTANA, M.S.M.; FERRETI, A.R.; TOLETO FILHO, D.V.; ÁRBOCZ, G.F.; SHIMIDT, H.A. \& GUARDIA, J.F.C. Espécies arbóreas nativas do estado de São Paulo recomendadas para reflorestamentos. In: CRESTANA, M.S.M.; FERRETI, A.R.; TOLETO FILHO, D.V.; ÁRBOCZ, G.F.; SHIMIDT, H.A. \& GUARDIA, J.F.C. Florestas: Sistemas de recuperação com essências nativas, produção de mudas e legislação. São Paulo, Imprensa Oficial, 2006. p.49-84.

CRUZ, C.D. Programa GENES - Aplicativo computacional em genética e estatística. Viçosa, MG, Universidade Federal de Viçosa, 1997. 442p.

CRUZ, C.D. \& REGAZZI, A.J. Modelos biométricos aplicados ao melhoramento genético. Viçosa, MG, Universidade Federal de Viçosa, 1994. p.390.

CUENCA, G.; ANDRADE, Z. \& ESCALANTE, G. Diversity of glomalean spores from natural, disturbed and revegetated communities growing on nutrient-poor tropical soils. Soil Biol. Biochem., 30:711-719, 1998.

DOUDS, D.D. \& MILLNER, P.D. Biodiversity of arbuscular mycorrhizal fungi in agroecossistemas. Agric. Ecosyst. Environ., 74:77-93, 1999.

FELDMANN, F. Mycorrhizal situation of native trees in the Brazilian tropical ecosystems Varzea, Igapo, and Terra Firme. In: LIEBEREI, R.; VOß, K. \& BRÜGMANN, C., eds. Hamburg, Tropische Nutzpflanzen, 1994. p.70-77.

FELDMANN, F.; SILVA JUNIOR, J.P. \& LIEBEREI, R. AMF spore community composition at natural and agricultural sites in Central Amazonia - a long term study. In: LIEBEREI, R.; BIANCHI, H.K.; BOEHM, V. \& REISDORFF, C., eds. Neotropical ecosystems. Hamburg, 2000. Proceedings of the German-Brazilian Workshop. Geesthacht, GKSS, 2002. p.669-682. 
FRANKE-SNYDER, M.; DOUDS JR, D.D.; GALVEZ, L.; PHILLIPS, J.G.; WAGONER, P.; DRINKWATER, L. \& MORTON, J.B. Diversity of communities of arbuscular mycorrhizal (AM) fungi present in convencional versus low-input agricultural sites in eastern Pennsylvania, USA. Appl. Soil Ecol., 16:35-48, 2001.

GERDEMANN, J.W. \& NICOLSON, T.H. Spores of mycorrhizal endogone species extracted from soil by wit sieving and decanting. Trans. Br. Mycol. Soc., 46:235-244, 1963.

GRACE, C. \& STRIBLEY, D.P. A safer produce for roution staining of vesicular-arbuscular mycorrhizal fungi. Mycol. Res., 95:1160-1162, 1991.

GUADARRAMA, P. \& ÁLVAREZ-SÁNCHES, F.J. Abundance of arbuscular mycorrhizal fungi spores in different environments in a tropical rain forest. Mycorrhiza, 8:267-270, 1999.

HAMMER, O.; HARPER, D.A.T. \& RYAN, P.D. PAST: Paleontological Statistics Software Package for Education and Data Analysis. Palaeontol. Electr., 4:1-9,2001.

INTERNATIONAL CULTURE COLLECTION OF ARBUSCU. LAR AND VESICULAR-ARBUSCULAR MYCORRHIZAL FUNGI - INVAM. West Virginia University. Disponível em: http:// invam.caf.wva.edu. Acesso em fev. de 2009.

JANSA, J.; MOZAFAR, A.; ANKEN, T.; RUH, R.; SANDERS, I.R. \& FROSSARD, E. Diversity and structure of AMF communities as affected by tillage in a temperate soil. Mycorrhiza, 12:225-234, 2002

JOHNSON, N.C. \& PFLEGER, F.L. Vesicular-arbuscular mycorrhizae and cultural stresses. In: BETHLENFALVAY, G.J. \& LINDERMAN, R.G., eds. Mycorrhizae in sustainable agriculture. Madison, ASA, 1992. p.71-97.

KLIRONOMOS, J.N.; McCUNE, J.; HART, M. \& NEVILLE, J. The influence of arbuscular mycorrhizae on the relationship between plant diversity and productivity. Ecol. Letters, 3:137-141, 2000

KOSKE, R.E. \& GEMMA, J.N. A modified procedure for staining roots to detect VA mycorrhizas. Mycol. Res., 92:486-488, 1989.

KUYPER, T.W.; CARDOSO, I.M.; ONGUENE, N.A. \& van NOORDWIJK, M.M. Managing mycorrhiza in tropical multispecies agroecosystems. In: van NOORDWIJK, M.; CADISCH, G. \& ONG, C.K., ed. Below-ground interactions in tropical agroecosystems: Concepts and models with multiple plant components. Wallingford, CABI Publishing, 2004. p.243-258.

MANSON, P.A.; MUSOKO, M.O. \& LAST, F.T. Short-term changes in vesicular-arbuscular mycorrhizal spore populations in Terminalia Plantations in Cameroon. In: READ, D.J.; LEWIS, D.H.; FITTER, A.H. \& ALEXANDER, I.J., eds. Mycorrhizas in ecossystems. Wallingford, CABI Publishing, 1982. p.261-267.

MATHIMARAN, N.; RUH, R.; JAMA, B.; VERCHOT, L.; FROSSARD, E. \& JANSA, J. Impact of agricultura management on arbuscular mycorrhizal fungal communities in Kenyan Ferralsol. Agric. Ecosyst. Environ., 119:22-32, 2007.

MIRANDA, J.C.C. \& MIRANDA, L.N. Micorriza arbuscular. In: VARGAS, M.A. \& HUNGRIA, M., eds. Biologia dos solos dos Cerrados. Planaltina, Embrapa-CPAC, 1997. p.69-123.
MIRANDA, J.C.C.; VILELA, L. \& MIRANDA, L.N. Dinâmica e contribuição da micorriza arbuscular em sistem as de produção com rotação de culturas. Pesq. Agropec. Bras., 40:1005-1014, 2005.

MIRANDA, J.C.C. Cerrado: Micorriza arbuscular, ocorrência e manejo. Planaltina, Embrapa Cerrados, 2008. p.169.

MORTON, J.B. Problems and solutions for the integration of Glomalean taxonomy, systematic biology, and the study of endomycorrhizal phenomena. Mycorrhiza, 2:97-109, 1993.

MOREIRA, F.M.S. \& SIQUEIRA, J.O. Microbiologia e bioquímica do solo. 2.ed. Lavras, Universidade Federal de Lavras, 2006. 729 p.

NICOLSON, T.H. Vesicular-arbuscular mycorrhiza: A universal plant symbiosis. Sci. Progress, 55:561-581, 1967.

ODUM, E.P. Ecologia. São Paulo, Guanabara, 1988. 450p.

OEHL, F.; SIEVERDING, E.; INECHEIN, K.; MÄDER P.; BOLLER, T. \& WIEMKEN, A. Impact of land use intensity on the species diversity of arbuscular mycorrhizal fungi in agroecosystems of Central Europe. Appl. Environ. Microbiol., 69:2816-2824, 2004

PICONE, C. Diversity and abundance of Arbuscular Mycorrhizal. Fungus spores in Tropical Forest and Pasture. Biotropica, 32:734-750, 2000.

PIROZYNSKI, K.A. Interactions between fungi and plants through the ages. Can. J. Bot., 59:1824-1827, 1981.

REDECKER, D.; KODNER, R. \& GRAHAM, L.E. Glomalean fungi from the Ordovician. Science, 289:1920-1921, 2000.

SAEG Sistema para Análises Estatísticas, Versão 9.1. Viçosa, MG, Fundação Arthur Bernardes/UFV, 2007.

SANTOS, E.V.; MARTINS, R.A. \& FERREIRA, I.M. O processo de ocupação do bioma cerrado e a degradação do subsistema vereda no sudeste de Goiás. In: SIMPÓSIO BRASILEIRO DE GEOGRAFIA FÍSICA APLICADA, 13., Viçosa, MG, 2009. Anais... Viçosa, MG, Universidade Federal de Viçosa, 2009. p.1-20. Disponível em <www.geo.ufv.br/simposio/ simposio/trabalhos/trabalhos.../eixo2/010.pdf $>$. Acesso em 16 de maio de 2010 .

SCHENCK, N.C. \& PÉREZ, Y. Manual for the identification of VA mycorrhizal fungi. 3.ed. Gainesville, Sinergistic Publications, 1990.

SCHENCK, N.C.; SIQUEIRA, J.O. \& OLIVEIRA, E. Changes in the incidence of VA mycorrhizal fungi with changes in ecosystems In: VANCURA, V. \& KUNC, F., eds. Interelationships between microorganisms and plant in soil. Prague, Elsevier, 1989. p.125-129.

SIEVERDING, E. Ecology of VAM fungi in tropical agrosystems. Agric. Ecosyst. Environ., 29:369-390, 1990.

SILVA JUNIOR, J.P. \& CARDOSO, E.J.B.N. Micorriza arbuscular em cupuaçu e pupunha cultivados em sistema agroflorestal e em monocultivo na Amazônia Central. Pesq. Agropec. Bras., 41:819-825 2006. 
SIQUEIRA, J.O.; MOREIRA, F.M.S.; GRISI, B.M.; HUNGRIA, M. \& ARAUJO, R.S. Microrganismos e processos biológicos do solo: Perspectiva ambiental. Brasília, Embrapa, 1994. p.142.

SIQUEIRA, J.O.; SOUSA, C.R.F.; SANTOS, J.G.D.; SCHNEIDER, J. \& CARNEIRO, M.A.C. Micorrízas e degradação do solo: Caracterização, efeitos e ação recuperadora. In: CERETTA, C.A.; SILVA, L.S. \& REICHERT, J.M., eds. Tópicos em ciência do solo. Viçosa, MG, Sociedade Brasileira de Ciência do Solo, 2007. v.5. p.219-305.

SOUZA, D.M.G. \& LOBATO, E. Cerrado: Correção do solo e adubação. 2.ed. Brasília, Embrapa Cerrados, 2004. p.416.

STADDON, P.L.; THOMPSON, K.; JAKOBSEN, I.; GRIME, J.P.; ASKEW, A.P. \& FITTER, A.H. Mycorrhizal fungi abundance as affected by long-term climatic manipulations in the field. Global Change Biol., 9:186-194, 2003.

STÜRMER S.L. \& SIQUEIRA, J.O. Diversidade de fungos micorrízicos arbusculares em ecossistemas brasileiros. In: MOREIRA, F.M.S.; SIQUEIRA, J.O. \& BRUSSAARD, L., eds. Biodiversidade do solo em ecossistemas brasileiros. Lavras, Universidade Federal de Lavras, 2008. p.537-583.
SYLVIA, D.M. \& WILLIAMS, S.E. Vesicular arbuscular mycorrhizae and environamental stress. In: BETHLENFALVAY, G.J. \& LINDERMAN, R.G., eds. Mycorrhizae in sustainable agriculture. Madison, American Society of Agronomy, 1992. p.101-124.

THOMPSON, J.P. Decline of vesicular-arbuscular mycorrhizae in long fallow disorder of field crops and its expression in phosphorus deficiency of sunflower. Austr. J. Agric. Res., 38:847-867, 1987.

TRESEDER, K. \& CROSS, A. global distribution of arbuscular mycorrhizal fungi. Ecosystems, 9:305-316, 2006.

van der HEIJDEN, M.G.A.; BOLLER, T.; WIENKEN, A. \& SANDERS, I.R. Different arbuscular mycorrhizal fungal species are potential determinants of plant community structure. Ecology, 79:2082-2091, 1998. 